\title{
A Legendre-Gauss collocation method for neutral functional-differential equations with proportional delays
}

\author{
Ali H Bhrawy ${ }^{1,2^{*}}$, Laila M Assas ${ }^{1,3}$, Emran Tohidi ${ }^{4}$ and Mohammed A Alghamdi ${ }^{1}$
}

${ }^{*}$ Correspondence:

alibhrawy@yahoo.co.uk

${ }^{1}$ Department of Mathematics,

Faculty of Science, King Abdulaziz

University, Jeddah, 21589, Saudi

Arabia

${ }^{2}$ Department of Mathematics,

Faculty of Science, Beni-Suef

University, Beni-Suef, Egypt

Full list of author information is

available at the end of the article

\begin{abstract}
In this paper, we present a unified framework for analyzing the spectral collocation method for neutral functional-differential equations with proportional delays using shifted Legendre polynomials. The proposed collocation technique is based on shifted Legendre-Gauss quadrature nodes as collocation knots. Error analysis and stability of the proposed algorithm are theoretically investigated under several mild conditions. The accuracy of the proposed method has been compared with a variational iteration method, a one-leg $\theta$-method, a particular Runge-Kutta method, and a reproducing kernel Hilbert space method. Numerical results show that the proposed methods are of high accuracy and are efficient for solving such an equation. Also, the results demonstrate that the proposed method is a powerful algorithm for solving other delay differential equations.
\end{abstract}

Keywords: delay differential equations; neutral functional-differential equations; proportional delays; spectral method; Legendre-Gauss quadrature

\section{Introduction}

One of the fundamental classes of delay differential equations (DDEs) is that of neutral functional-differential equations (NFDEs) with proportional delays. Such equations arise in many areas of science and engineering and play an important role in the modeling of real-life phenomena in other fields of science $(c f$. [1-5]). For these reasons, NFDEs have received much attention in the last decades. The principal difficulty in studying DDEs lies in their special transcendental nature. Obviously, most of NFDEs cannot be solved by the well-known exact methods. Therefore, it is highly desirable to design accurate numerical approaches to approximate the solutions of NFDEs.

The theory of DDEs with multiple delays has been analyzed by many authors, and we briefly review some of them. In [6] Jackiewicz and Lo proposed and developed the Adams predictor-corrector method for the numerical solution of NFDEs. In [7], the authors investigated the Adomian decomposition method for solving a special class of DDEs and established the convergence of this approach.

Some analytical and numerical solutions of a family of DDEs were presented in [8]. The authors of [9] investigated the Runge-Kutta (RK) method for NFDEs with different proportional delays and proved the stability of RK for this equation. Wang and Li [10] proposed the one-leg-methods for solving nonlinear neutral functional differential equations. 
We also refer to the articles [11-13] and the references therein for applying the waveform relaxation method to solve neutral type functional-differential systems.

Over the years, it was found that the spectral methods were a valid method to obtain approximations for differential equations. The solution of a DDE globally depends on its history due to the delay variable, so a global spectral method could be a good candidate for numerical DDEs. In this direction, Ishiwata and Muroya [14] proposed the rational approximation algorithm based on Legendre polynomials and Ishiwata et al. [15] developed the collocation approximation for solving DDEs. Meanwhile, in [16] the authors discussed the tau approach for solving a class of NFDEs, and the solution was expanded by a shifted Legendre polynomial, and the unknown expansion coefficients were obtained by a segmented Lanczos-tau formulation. The authors of [17] proposed a Bernoulli operational matrix method for solving a generalized pantograph equation. Wang and Wang [18] proposed and analyzed the Legendre collocation algorithm for nonlinear DDEs with variable delay. The theory and the numerical results given in [18] and [19] explained that the proposed collocation approximations converge exponentially when the data in the given DDEs are smooth. Recently, Ali et al. [20] implemented a spectral Legendre approach for solving pantograph-type differential and integral equations and studied the error analysis of the method. More recently, the work of Trif [21] discussed the application of the tau method based on the operational matrix of Chebyshev polynomials for solving DDEs of pantograph type.

Our fundamental goal of this paper is to develop a suitable way to approximate the neutral functional-differential equations with proportional delays on the interval $[0, T]$ by using the shifted Legendre polynomials. In other words, we propose the spectral shifted Legendre-Gauss collocation (SLC) method to find the solution $u_{N}(x)$. For suitable collocation points, we use the $(N-m+1)$ nodes of the shifted Legendre-Gauss interpolation on the interval $[0, T]$. These equations together with $m$ initial conditions generate $(N+1)$ algebraic equations which can be solved. It should be noted that the basic requirement for using any spectral base (e.g., Legendre polynomials) is the smoothness of the solution of the considered problem. This may be guaranteed by the smoothness of known functions in the neutral equation. By these assumptions (i.e., being smooth), exponential convergence behavior of spectral approximations is exhibited in any test problem.

The paper is organized as follows. Section 2 is devoted to preliminaries needed hereafter. In Section 3, we design the shifted Legendre-Gauss collocation technique for NFDEs with proportional delays. The error analysis and stability of the solution are provided in Section 4 under several mild conditions. In Section 5, we present some numerical results demonstrating the efficiency of the suggested numerical algorithm. Concluding remarks are given in Section 6.

\section{Preliminaries}

Let $P_{i}(x)$ be the standard Legendre polynomial of degree $i$, then we have that

$$
P_{i}(-x)=(-1)^{i} P_{i}(x), \quad P_{i}(-1)=(-1)^{i}, \quad P_{i}(1)=1 .
$$

If $\alpha>-1, \beta>-1$ and $P_{k}^{(\alpha, \beta)}(x)$ is the $k$ th degree of the Jacobi polynomial, then for an integer $m$, the $m$ th-order derivative of Legendre polynomials is

$$
D^{m} P_{k}(x)=2^{-m} \frac{\Gamma(m+k+1)}{\Gamma(k+1)} P_{k-m}^{(m, m)}(x) .
$$


Let $w^{(\alpha, \beta)}(x)=(1-x)^{\alpha}(1+x)^{\beta}$, then we define the weighted space $\mathrm{E}_{w^{(\alpha, \beta)}}^{2}(-1,1)$ as usual, equipped with the following inner product and norm:

$$
(u, v)_{w^{(\alpha, \beta)}}=\int_{-1}^{1} u(x) v(x) w^{(\alpha, \beta)}(x) d x, \quad\|v\|_{w^{(\alpha, \beta)}}=(v, v)_{w^{(\alpha, \beta)}}^{\frac{1}{2}} .
$$

The set of Legendre polynomials forms a complete $\mathrm{七}^{2}(-1,1)$-orthogonal system, and

$$
\left\|P_{i}(x)\right\|^{2}=h_{i}=\frac{2}{2 i+1} .
$$

Let $T>0$, then the shifted Legendre polynomial of degree $k$ on the interval $(0, T)$ is defined by $L_{T, k}(x)=P_{k}\left(\frac{2 x}{T}-1\right)$. Thus $L_{T, k}(x)$ can be obtained by

$$
(k+1) L_{T, k+1}(x)=(2 k+1)\left(\frac{2 x}{T}-1\right) L_{T, k}(x)-k L_{T, k-1}(x), \quad k=1,2, \ldots
$$

With the aid of the standard Legendre polynomials, we get

$$
L_{T, k}(0)=(-1)^{k}, \quad D^{q} L_{T, k}(0)=\frac{(-1)^{k-q}(k+q) !}{T^{q}(k-q) ! q !}, \quad D^{q} L_{T, k}(T)=\frac{(k+q) !}{T^{q}(k-q) ! q !} .
$$

Next, we define the space $\iota^{2}(0, T)$ in the usual way, with the following inner product and norm:

$$
(u, v)=\int_{0}^{T} u(x) v(x) d x, \quad\|u\|=(u, u)^{1 / 2} .
$$

The set of shifted Legendre polynomials is a complete $亡^{2}(0, T)$-orthogonal system. Moreover, due to (3), we have

$$
\left\|L_{T, k}\right\|^{2}=\left(\frac{T}{2}\right) h_{k}=h_{T, k}
$$

\section{Shifted Legendre-Gauss collocation method}

In this section, we develop a spectral Legendre-Gauss collocation approach to analyze the following NFDEs with proportional delays:

$$
\left(u(x)+a(x) u\left(\gamma_{m} x\right)\right)^{(m)}=\beta u(x)+\sum_{n=0}^{m-1} b_{n}(x) u^{(n)}\left(\gamma_{n} x\right)+f(x), \quad x \geq 0,
$$

with the initial conditions

$$
u^{(n)}(0)=\lambda_{n}, \quad n=0,1, \ldots, m-1 .
$$

Here, $a(x)$ and $b_{n}(x)(n=0,1, \ldots, m-1)$ are given analytical functions, and $\gamma_{n}, \beta, \lambda_{n}$ are constants with $0<\gamma_{n}<1(n=0,1, \ldots, m)$.

Now we introduce the Legendre-Gauss quadratures in two different intervals $(-1,1)$ and $(0, T)$ that will be used in the sequel. Denote by $x_{N, j}\left(x_{T, N, j}\right), 0 \leq j \leq N$, and $\varpi_{N, j}\left(\varpi_{T, N, j}\right)$, 
$0 \leq i \leq N$, the nodes and Christoffel numbers of the standard (shifted) Legendre-GaussLobatto quadratures on the intervals $(-1,1),(0, T)$, respectively. Then one can clearly deduce that [22]

$$
\begin{array}{ll}
x_{T, N, j}=\frac{T}{2}\left(x_{N, j}+1\right), & 0 \leq j \leq N, \\
\varpi_{T, N, j}=\left(\frac{T}{2}\right) \varpi_{N, j}, & 0 \leq j \leq N,
\end{array}
$$

and if $S_{N}(0, T)$ denotes the set of all polynomials of degree at most $N$, then it follows that for any $\phi \in S_{2 N+1}(0, T)(c f .[23])$,

$$
\begin{aligned}
\int_{0}^{T} \phi(x) d x & =\left(\frac{T}{2}\right) \int_{-1}^{1} \phi\left(\frac{T}{2}(x+1)\right) d x \\
& =\left(\frac{T}{2}\right) \sum_{j=0}^{N} \varpi_{N, j} \phi\left(\frac{T}{2}\left(x_{N, j}+1\right)\right)=\sum_{j=0}^{N} \varpi_{T, N, j} \phi\left(x_{T, N, j}\right) .
\end{aligned}
$$

We define the discrete inner product and norm as follows:

$$
(u, v)_{N}=\sum_{k=0}^{N} u\left(x_{T, N, k}\right) v\left(x_{T, N, k}\right) \varpi_{N, k}, \quad\|u\|_{N}=\sqrt{(u, u)_{N}} .
$$

Obviously,

$$
(u, v)_{N}=(u, v) \quad \forall u v \in S_{2 N-1} .
$$

Thus, for any $u \in S_{N}(0, T)$, the norms $\|u\|_{N}$ and $\|u\|$ coincide. We set

$$
S_{N}(0, T)=\operatorname{span}\left\{L_{T, 0}(x), L_{T, 1}(x), \ldots, L_{T, N}(x)\right\} .
$$

The following relation for the $q$ th derivative of shifted Legendre polynomials $L_{T, k}(x)$ will be needed for our main results [24]:

$$
L_{T, k}^{(q)}(x)=\sum_{\substack{i=0 \\(k+i) \text { even }}}^{k-q} C_{q}(k, i) L_{T, i}(x)
$$

where

$$
C_{q}(k, i)=\frac{2^{q-1}(2 i+1) \Gamma\left(\frac{1}{2}(q+k-i)\right) \Gamma\left(\frac{1}{2}(q+k+i+1)\right)}{T^{q} \Gamma(q) \Gamma\left(\frac{1}{2}(2-q+k-i)\right) \Gamma\left(\frac{1}{2}(3-q+k+i)\right)} .
$$

In virtue of (13), the high-order derivative of shifted Legendre polynomials with proportional delay can be written as

$$
L_{T, k}^{(q)}\left(\gamma_{m} x\right)=\sum_{\substack{i=0 \\(k+i) \text { even }}}^{k-q} \gamma_{m}^{q} C_{q}(k, i) L_{T, i}\left(\gamma_{m} x\right)
$$

where $C_{q}(k, i)$ is defined in (14). 
The shifted Legendre-Gauss collocation method for solving (7) and (8) is to seek $u_{N}(x) \in$ $S_{N}(0, T)$ such that

$$
\begin{aligned}
& \left(u\left(x_{T, N, k}\right)+a\left(x_{T, N, k}\right) u\left(\gamma_{m} x_{T, N, k}\right)\right)^{(m)}=\beta u\left(x_{T, N, k}\right)+\sum_{n=0}^{m-1} b_{n}\left(x_{T, N, k}\right) u^{(n)}\left(\gamma_{n} x_{T, N, k}\right) \\
& +f\left(x_{T, N, k}\right), \quad k=0,1, \ldots, N-m, \\
& u^{(n)}(0)=\lambda_{n}, \quad n=0,1, \ldots, m-1 .
\end{aligned}
$$

Now, we derive an efficient algorithm for tackling (7) and (8). Let us expand the numerical approximation in terms of shifted Legendre polynomials

$$
u_{N}(x)=\sum_{h=0}^{N} a_{h} L_{T, h}(x), \quad \mathbf{a}=\left(a_{0}, a_{1}, \ldots, a_{N}\right)^{T} .
$$

We first approximate $u(x)$ and $u^{n}(x)$ as Eq. (17). By substituting these approximations in Eq. (7), we get

$$
\begin{aligned}
& \left(\sum_{h=0}^{N} a_{h} L_{T, h}(x)+a(x) \sum_{h=0}^{N} a_{h} L_{T, h}\left(\gamma_{m} x\right)\right)^{(m)} \\
& \quad=\beta \sum_{h=0}^{N} a_{h} L_{T, h}(x)+\sum_{h=0}^{m-1} \sum_{h=0}^{N} b_{h}(x) a_{h} D^{(n)} L_{T, h}\left(\gamma_{h} x\right)+f(x) .
\end{aligned}
$$

Then, by virtue of (13), we deduce that

$$
\begin{aligned}
& \left(\sum_{h=0}^{N} a_{h} L_{T, h}(x)+a(x) \sum_{h=0}^{N} a_{h} L_{T, h}\left(\gamma_{m} x\right)\right)^{(m)} \\
& \quad=\beta \sum_{h=0}^{N} a_{h} L_{T, h}(x)+\sum_{n=0}^{m-1} \sum_{h=0}^{N} \sum_{f=0}^{h-q}\left(\gamma_{n}\right)^{n} b_{n}(x) a_{h} C_{q}(h, f) L_{T, f}\left(\gamma_{n} x\right)+f(x) .
\end{aligned}
$$

Also, by substituting Eq. (17) in Eq. (8), we obtain

$$
\sum_{h=0}^{N} a_{h} D^{(n)} L_{T, h}(0)=\lambda_{n}, \quad n=0,1, \ldots, m-1 .
$$

To find the solution $u_{N}(x)$, we collocate Eq. (19). Using (13) and (15), at the $(N-m+1)$ shifted Legendre roots, yields

$$
\begin{aligned}
& \left(\sum_{h=0}^{N} a_{h} L_{T, h}\left(x_{T, N, k}\right)+a\left(x_{T, N, k}\right) \sum_{h=0}^{N} a_{h} L_{T, h}\left(\gamma_{m} x_{T, N, k}\right)\right)^{(m)} \\
& =\beta \sum_{h=0}^{N} a_{h} L_{T, h}\left(x_{T, N, k}\right) \\
& \quad+\sum_{n=0}^{m-1} \sum_{h=0}^{N} \sum_{f=0}^{h-q}\left(\gamma_{n}\right)^{n} b_{n}\left(x_{T, N, k}\right) a_{h} C_{q}(h, f) L_{T, f}\left(\gamma_{n} x_{T, N, k}\right)+f\left(x_{T, N, k}\right) .
\end{aligned}
$$


Next, Eq. (20), after using (4), can be written as

$$
\sum_{h=0}^{N}(-1)^{h-n} a_{h} \frac{(h+n) !}{T^{n}(h-n) ! n !}=\lambda_{n}, \quad n=0,1, \ldots, m-1 .
$$

Thus Eq. (21) with relation (22) generate $(N+1)$ of a set of algebraic equations which can be solved for the unknown coefficients $a_{j}, j=0,1,2, \ldots, N$, by using any standard solver technique.

\section{Error analysis and stability of the solution}

This section is divided into two subsections. The first subsection is related to presenting a bound for the error of the proposed method; meanwhile, in the second subsection, the stability of the numerical solution is investigated briefly.

\subsection{Error bound of the method}

In this part, the error bound of the method will be provided under several mild conditions such as solution boundedness of the main neutral differential equation. However, some definitions and lemmas should be provided for clarifying the main theorem of this subsection.

Definition 4.1 A function, $\xi:[-1,1] \longrightarrow \Re$ belongs to the Sobolev space $W^{m, p}$, if its $j$ th weak derivative, $\xi^{(j)}$, lies in $L^{p}[-1,1]$ for all $0 \leq j \leq m$ with the norm

$$
\|\xi\|_{W^{m, p}}=\sum_{j=0}^{m}\left\|\xi^{(j)}\right\|_{L^{p}}
$$

where $\|\xi\|_{L^{p}}$ denotes the usual Lebesgue norm,

$$
\|\xi\|_{L^{p}}=\left(\int_{-1}^{1}\|\xi(x)\|^{p} d x\right)^{\frac{1}{p}}
$$

and $\|\xi(x)\|$ stands for any finite dimensional norm in $\Re^{n}$.

Lemma 4.1 Given a function $\xi \in W^{m, \infty}, x \in[-1,1]$, there exists a polynomial $u_{N}(x)$ of degree less than or equal to $N$ such that

$$
\left\|\xi(x)-u_{N}(x)\right\|_{L^{\infty}} \leq C C_{0} N^{-m}, \quad \forall x \in[-1,1]
$$

where $C$ is a constant independent of $N, m$ is the order of smoothness of $\xi$, and $C_{0}=$ $\|\xi\|_{W^{m}, \infty}$. Here, $u_{N}(x)$ with the smallest norm $\left\|\xi(x)-u_{N}(x)\right\|_{L^{\infty}}$ is called the Nth order best polynomial approximation of $\xi(x)$ in the norm of $L^{\infty}$.

Note that if $\xi \in C^{\infty}$, then $m=\infty$. This implies that $u_{N}(x)$ converges to $\xi$ at a spectral rate, i.e., it is faster than any given polynomial rate. Moreover, we denote the set of continuous functions in a linear space on $[0, T]$ by $C[0, T]$ and the uniform norm in $C[0, T]$ by

$$
\|f\|_{\infty}=\max _{0 \leq x \leq t}|f(x)|, \quad \forall f \in C[0, T]
$$


Again, we consider Eq. (7) with the initial conditions (8). For clarity of presentation, we assume that $m=1$. A similar procedure can be applied for higher values of $m$. Therefore, (7)-(8) can be written as follows:

$$
\left(u(x)+a(x) u\left(\gamma_{1} x\right)\right)^{\prime}=\beta u(x)+b_{0}(x) u\left(\gamma_{0} x\right)+f(x), \quad u(0)=\lambda_{0}
$$

Integrating the aforementioned equation in the interval $[0, x]$ yields

$$
u(x)+a(x) u\left(\gamma_{1} x\right)-\left(\lambda_{0}+a(0) \lambda_{0}\right)=\int_{0}^{x}\left(\beta u(\varepsilon)+b_{0}(\varepsilon) u\left(\gamma_{0} \varepsilon\right)+f(\varepsilon)\right) d \varepsilon
$$

Accordingly, we can rewrite the above-obtained equation in the following form:

$$
u(x)+a(x) u\left(\gamma_{1} x\right)=g(x)+\beta \int_{0}^{x} u(\varepsilon) d \varepsilon+\int_{0}^{\gamma_{0} x} \hat{b}_{0}(\theta) u(\theta) d \theta
$$

where $g(x)=\left(\lambda_{0}+a(0) \lambda_{0}\right)+\int_{0}^{x} f(\varepsilon) d \varepsilon$ and $\hat{b}_{0}(\theta)=\frac{1}{\gamma_{0}} b_{0}\left(\frac{\theta}{\gamma_{0}}\right)$.

In the following theorem, we show that the approximate solution which was expressed in terms of Legendre polynomials converges to the exact solution under several mild conditions.

Theorem 4.2 Consider Eq. (23) again. Assume $u(x)$ and $u_{N}(x)$ are the exact and approximate solutions of (23). Also, let the approximations of $a(x), g(x)$, and $\hat{b}_{0}(x)$ be $a_{N}(x)$, $g_{N}(x)$, and $\hat{b}_{0, N}(x)$, respectively. Moreover, suppose that $\|a(x)\|_{\infty} \leq A,\left\|u_{N}(x)\right\|_{\infty} \leq U_{N}$, $\left\|\hat{b}_{0}(x)\right\|_{\infty} \leq B$, where $x \in[0, T]$. Then $\lim _{N \rightarrow \infty} u_{N}(x)=u(x)$ under the condition $A+|\beta| T+$ $T B \ll 1$.

Proof Since the known functions $a(x), g(x)$, and $\hat{b}_{0}(x)$ are approximated in terms of shifted Legendre polynomials, then the obtained solution is an approximated polynomial in the form of $u_{N}(x)$. Our aim is to find an upper bound for the associated error between $u(x)$ and $u_{N}(x)$ for (23). We rewrite (23) in the following form:

$$
u(x)=-a(x) u\left(\gamma_{1} x\right)+g(x)+\beta \int_{0}^{x} u(\varepsilon) d \varepsilon+\int_{0}^{\gamma_{0} x} \hat{b}_{0}(\theta) u(\theta) d \theta
$$

According to the assumptions, one reaches the following equation:

$$
u_{N}(x)=-a_{N}(x) u_{N}\left(\gamma_{1} x\right)+g_{N}(x)+\beta \int_{0}^{x} u_{N}(\varepsilon) d \varepsilon+\int_{0}^{\gamma_{0} x} \hat{b}_{0, N}(\theta) u_{N}(\theta) d \theta
$$

Subtraction of the above equations yields

$$
\begin{aligned}
& \left\|u(x)-u_{N}(x)\right\|_{\infty} \\
& =\|-a(x) u\left(\gamma_{1} x\right)+a_{N}(x) u_{N}\left(\gamma_{1} x\right)+g(x)-g_{N}(x)+\beta \int_{0}^{x} u(t) d t \\
& \quad-\beta \int_{0}^{x} u_{N}(\varepsilon) d \varepsilon+\int_{0}^{\gamma_{0} x} \hat{b}_{0}(\theta) u(\theta) d \theta-\int_{0}^{\gamma_{0} x} \hat{b}_{0, N}(\theta) u_{N}(\theta) d \theta \|_{\infty} .
\end{aligned}
$$


According to the trigonometric inequality, we have

$$
\begin{aligned}
\left\|u(x)-u_{N}(x)\right\|_{\infty} \leq & \left\|-a(x) u\left(\gamma_{1} x\right)+a_{N}(x) u_{N}\left(\gamma_{1} x\right)\right\|_{\infty}+\left\|g(x)-g_{N}(x)\right\|_{\infty} \\
& +\left\|\beta \int_{0}^{x} u(\varepsilon) d \varepsilon-\beta \int_{0}^{x} u_{N}(\varepsilon) d \varepsilon\right\|_{\infty} \\
& +\left\|\int_{0}^{\gamma_{0} x} \hat{b}_{0}(\theta) u(\theta) d \theta-\int_{0}^{\gamma_{0} x} \hat{b}_{0, N}(\theta) u_{N}(\theta) d \theta\right\|_{\infty} .
\end{aligned}
$$

Since $0 \leq \gamma_{0} \leq 1$ and $x \in[0, T]$, the above inequality reduces to a simple form as follows:

$$
\begin{aligned}
\left\|u(x)-u_{N}(x)\right\|_{\infty} \leq & \left\|a(x)\left(u\left(\gamma_{1} x\right)-u_{N}\left(\gamma_{1} x\right)\right)\right\|_{\infty}+\left\|\left(a(x)-a_{N}(x)\right) u_{N}\left(\gamma_{1} x\right)\right\|_{\infty} \\
& +\left\|g(x)-g_{N}(x)\right\|_{\infty}+T\left\|\beta\left(u(x)-u_{N}(x)\right)\right\|_{\infty} \\
& +T\left\|\hat{b}_{0}(x) u(x)-\hat{b}_{0, N}(x) u_{N}(x)\right\|_{\infty} .
\end{aligned}
$$

In other words,

$$
\begin{aligned}
\left\|u(x)-u_{N}(x)\right\|_{\infty} \leq & \left\|a(x)\left(u\left(\gamma_{1} x\right)-u_{N}\left(\gamma_{1} x\right)\right)\right\|_{\infty}+\left\|\left(a(x)-a_{N}(x)\right) u_{N}\left(\gamma_{1} x\right)\right\|_{\infty} \\
& +\left\|g(x)-g_{N}(x)\right\|_{\infty}+T\left\|\beta\left(u(x)-u_{N}(x)\right)\right\|_{\infty} \\
& +T\left\|\hat{b}_{0}(x)\left(u(x)-u_{N}(x)\right)\right\|_{\infty}+T\left\|\left(\hat{b}_{0}(x)-\hat{b}_{0, N}(x)\right) u_{N}(x)\right\|_{\infty} .
\end{aligned}
$$

Since $0 \leq \gamma_{1} \leq 1$, then $\lim _{N \rightarrow \infty}\left\|u(x)-u_{N}(x)\right\|_{\infty}=\lim _{N \rightarrow \infty}\left\|u\left(\gamma_{1} x\right)-u_{N}\left(\gamma_{1} x\right)\right\|_{\infty}$. Also, imposing the assumptions $\|a(x)\|_{\infty} \leq A,\left\|u_{N}(x)\right\|_{\infty} \leq U_{N},\left\|\hat{b}_{0}(x)\right\|_{\infty} \leq B$ to the above inequality yields

$$
\begin{aligned}
\left\|u(x)-u_{N}(x)\right\|_{\infty} \leq & A\left\|u(x)-u_{N}(x)\right\|_{\infty}+U_{N}\left\|a(x)-a_{N}(x)\right\|_{\infty} \\
& +\left\|g(x)-g_{N}(x)\right\|_{\infty}+|\beta| T\left\|u(x)-u_{N}(x)\right\|_{\infty} \\
& +T B\left\|u(x)-u_{N}(x)\right\|_{\infty}+T U_{N}\left\|\hat{b}_{0}(x)-\hat{b}_{0, N}(x)\right\|_{\infty} .
\end{aligned}
$$

For clarity of presentation, we rewrite the above inequality in the following form:

$$
\left\|u(x)-u_{N}(x)\right\|_{\infty} \leq \frac{U_{N}\left\|a(x)-a_{N}(x)\right\|_{\infty}+\left\|g(x)-g_{N}(x)\right\|_{\infty}+T U_{N}\left\|\hat{b}_{0}(x)-\hat{b}_{0, N}(x)\right\|_{\infty}}{1-A-|\beta| T-T B} .
$$

If $A+|\beta| T+T B \ll 1$, then $\lim _{N \rightarrow \infty}\left\|u(x)-u_{N}(x)\right\|_{\infty}=0$ (or $\lim _{N \rightarrow \infty} u_{N}(x)=u(x)$ ). This conclusion is made because of the smoothness of $a(x), g(x)$, and $\hat{b}_{0}(x)$. These assumptions imply that $\lim _{N \rightarrow \infty}\left\|a(x)-a_{N}(x)\right\|_{\infty}=0, \lim _{N \rightarrow \infty}\left\|g(x)-g_{N}(x)\right\|_{\infty}=0$, and $\lim _{N \rightarrow \infty} \| \hat{b}_{0}(x)-$ $\hat{b}_{0, N}(x) \|_{\infty}=0$ (see Lemma 4.1). This completes the proof.

\subsection{Stability of the solution}

Theorem 4.3 Let us consider $u(x) \in C[0, T]$ together with its Lagrange interpolation polynomial $I_{N} u(x)$ which is based on the shifted Gaussian points; then

$$
\left\|u-I_{N} u\right\|_{\infty} \leq\left(1+\Lambda_{N}\right)\left\|u-p^{*}\right\|_{\infty}
$$


where $p^{*}$ is the best approximation of $N$ th order of $u$ and

$$
\Lambda_{N}=\max _{-1 \leq x \leq 1} \lambda_{N}(x), \quad \lambda_{N}(x)=\sum_{j=0}^{N}\left|F_{j}(x)\right|
$$

are the Lebesgue constant and the Lebesgue function, respectively. Also, $F_{j}(x)$ stands for the jth Lagrange polynomial which is constructed by using shifted Gaussian points.

Proof Since $u(x) \in C[0, T]$, the best approximating polynomial for $u$, say $p^{*}$, exists and we immediately have

$$
\left\|u-I_{N} u\right\|_{\infty} \leq\left\|u-p^{*}\right\|_{\infty}+\left\|p^{*}-I_{N} u\right\|_{\infty} .
$$

However, both $p^{*}$ and $I_{N} u$ are $N$ th order polynomials

$$
\left\|p^{*}-I_{N} u\right\|_{\infty} \leq \Lambda_{N}\left\|u-p^{*}\right\|_{\infty}
$$

where

$$
\Lambda_{N}=\max _{-1 \leq x \leq 1} \lambda_{N}(x), \quad \lambda_{N}(x)=\sum_{j=0}^{N}\left|F_{j}(x)\right| .
$$

The Lebesgue function and the Lebesgue constant both depend only on the choice of interpolation nodes. The above theorem indicates that a good set of grid points is one which minimizes the Lebesgue constant for interpolation. We know that on equally spaced interpolation points, the Lebesgue constant blows up exponentially with the increasing of $N$ (as made apparent by the classical Runge phenomenon). On the contrary, the best possible Lebesgue constant among all distributions of $N$ prohibits only a logarithmic growth with $N$. Fortunately, the main families of Gaussian points (Gauss, Gauss-Radau, GaussLobatto) for the Legendre or Chebyshev weights have Lebesgue constants that grow logarithmically or sub-linearly with $N$.

Errors in the experimental measure of the data and computational issues such as rounding errors can impact on the accuracy of the interpolation. Assume that $u_{\epsilon}(x)$ represents a perturbed version of $u(x)$; i.e.,

$$
\left\|u-u_{\epsilon}\right\| \leq \epsilon
$$

the difference between the two polynomial representations is then

$$
\left\|I_{N} u-I_{N} u_{\epsilon}\right\| \leq \epsilon \Lambda_{N}
$$

Now we can deduce that for large $N$, Gaussian interpolation points with respect to equally spaced points have better stability, and for equally spaced points, an interpolation polynomial can become unstable for large $N$. 
Table 1 Absolute errors using SLC method at $N=17$ for Example 1

\begin{tabular}{|c|c|c|c|c|c|c|}
\hline \multirow[t]{2}{*}{$x$} & \multirow{2}{*}{$\begin{array}{l}\text { SLC method } \\
N=17\end{array}$} & \multirow{2}{*}{$\begin{array}{l}\text { RKHSM } \\
{[25]}\end{array}$} & \multicolumn{2}{|c|}{ VI method [26] } & \multirow{2}{*}{$\begin{array}{l}\text { One-leg } \theta \\
\text { method }[10,27]\end{array}$} & \multirow{2}{*}{$\begin{array}{l}\text { RKT } \\
\text { method [28] }\end{array}$} \\
\hline & & & $n=5$ & $n=6$ & & \\
\hline 0.1 & $4.27 \cdot 10^{-17}$ & $1.42 \cdot 10^{-4}$ & $2.63 \cdot 10^{-3}$ & $1.30 \cdot 10^{-3}$ & $4.65 \cdot 10^{-3}$ & $8.68 \cdot 10^{-4}$ \\
\hline 0.2 & $2.70 \cdot 10^{-17}$ & $1.17 \cdot 10^{-4}$ & $4.36 \cdot 10^{-3}$ & $2.14 \cdot 10^{-3}$ & $1.45 \cdot 10^{-2}$ & $1.49 \cdot 10^{-3}$ \\
\hline 0.3 & $5.94 \cdot 10^{-17}$ & $9.45 \cdot 10^{-4}$ & $5.40 \cdot 10^{-3}$ & $2.63 \cdot 10^{-3}$ & $2.57 \cdot 10^{-2}$ & $1.90 \cdot 10^{-3}$ \\
\hline 0.4 & $8.01 \cdot 10^{-17}$ & $7.59 \cdot 10^{-4}$ & $5.89 \cdot 10^{-3}$ & $2.84 \cdot 10^{-3}$ & $3.60 \cdot 10^{-2}$ & $2.16 \cdot 10^{-3}$ \\
\hline 0.5 & $8.27 \cdot 10^{-17}$ & $6.03 \cdot 10^{-4}$ & $5.96 \cdot 10^{-3}$ & $2.83 \cdot 10^{-3}$ & $4.43 \cdot 10^{-2}$ & $2.28 \cdot 10^{-3}$ \\
\hline 0.6 & $1.95 \cdot 10^{-16}$ & $4.73 \cdot 10^{-4}$ & $5.71 \cdot 10^{-3}$ & $2.67 \cdot 10^{-3}$ & $5.03 \cdot 10^{-2}$ & $2.31 \cdot 10^{-3}$ \\
\hline 0.7 & $1.56 \cdot 10^{-16}$ & $3.64 \cdot 10^{-4}$ & $5.23 \cdot 10^{-3}$ & $2.39 \cdot 10^{-3}$ & $5.37 \cdot 10^{-2}$ & $2.27 \cdot 10^{-3}$ \\
\hline 0.8 & $8.80 \cdot 10^{-17}$ & $2.75 \cdot 10^{-4}$ & $4.59 \cdot 10^{-3}$ & $2.04 \cdot 10^{-3}$ & $5.47 \cdot 10^{-2}$ & $2.17 \cdot 10^{-3}$ \\
\hline 0.9 & $1.03 \cdot 10^{-16}$ & $2.03 \cdot 10^{-4}$ & $3.84 \cdot 10^{-3}$ & $1.64 \cdot 10^{-3}$ & $5.35 \cdot 10^{-2}$ & $2.03 \cdot 10^{-3}$ \\
\hline 1.0 & $1.23 \cdot 10^{-16}$ & $1.43 \cdot 10^{-4}$ & $3.04 \cdot 10^{-3}$ & $1.22 \cdot 10^{-3}$ & $5.03 \cdot 10^{-2}$ & $1.86 \cdot 10^{-3}$ \\
\hline
\end{tabular}

\section{Numerical results}

To illustrate the effectiveness of the proposed method in the present paper, several test examples are carried out in this section. Comparisons of the results obtained by the present method with those obtained by other methods reveal that the present method is very effective and convenient. We consider the following examples.

Example 1 Consider the first-order neutral functional-differential equation with proportional delay used in [25]

$$
\left\{\begin{array}{l}
u^{\prime}(x)=-u(x)+0.1 u(0.8 x)+0.5 u^{\prime}(0.8 x)+(0.32 x-0.5) e^{-0.8 x}+e^{-x}, \quad x \geq 0 \\
u(0)=0
\end{array}\right.
$$

which has the exact solution $x e^{-x}$.

We apply the proposed shifted Legendre-Gauss collocation method at $t=1$ to (24). In Table 1, we compare our results with those obtained by a reproducing kernel Hilbert space method (RKHSM) [25], a variational iteration (VI) method [26], a one-leg $\theta$-method [10, 27], and a Runge-Kutta-type (RKT) method [28]. Numerical results of this problem show that the SLC method is more accurate than the presented methods in [10, 25-28]. The graph of the analytical solution and the approximate solution at $t=4$ for $N=15$ is displayed in Figure 1 to make it easer to compare with the analytical solution.

The logarithmic graph of absolute coefficients of shifted Legendre polynomials of a neutral functional-differential equation with proportional delay is shown in Figure 2, which shows that the method has exponential convergence rate. Absolute errors obtained by the SLC method with $N=17$ are plotted in Figure 3 .

Example 2 Consider the following first-order neutral functional-differential equation with proportional delay $[10,26,27,29,30]$ :

$$
\left\{\begin{array}{l}
u^{\prime}(x)=-u(x)+\frac{1}{2} u\left(\frac{x}{2}\right)+\frac{1}{2} u^{\prime}\left(\frac{x}{2}\right), \quad x \in[0,1] \\
u(0)=1
\end{array}\right.
$$

the analytic solution of the aforementioned problem is $u(x)=e^{-x}$. 


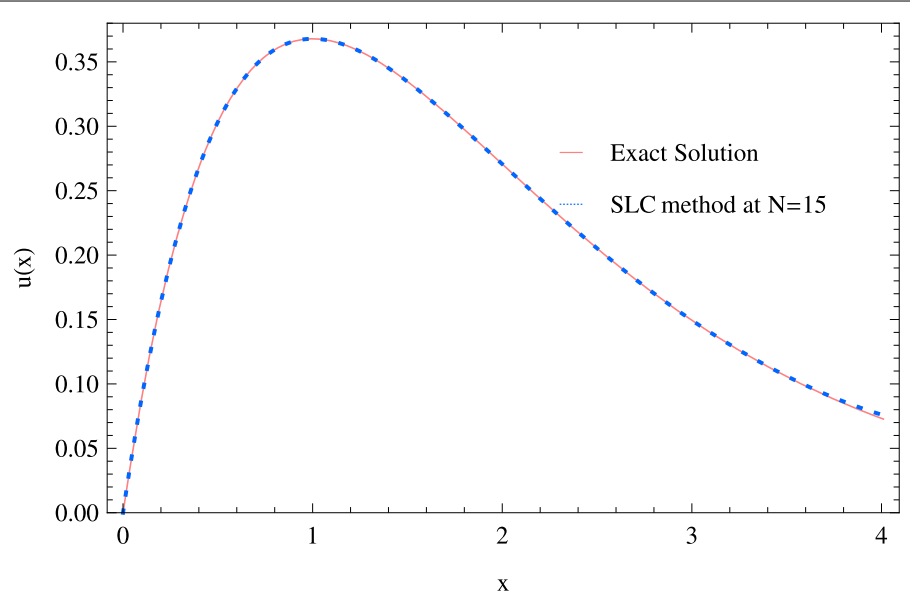

Figure 1 Graph of the exact solution and the approximate solution at $t=4$ and $N=15$ for Example 1 .

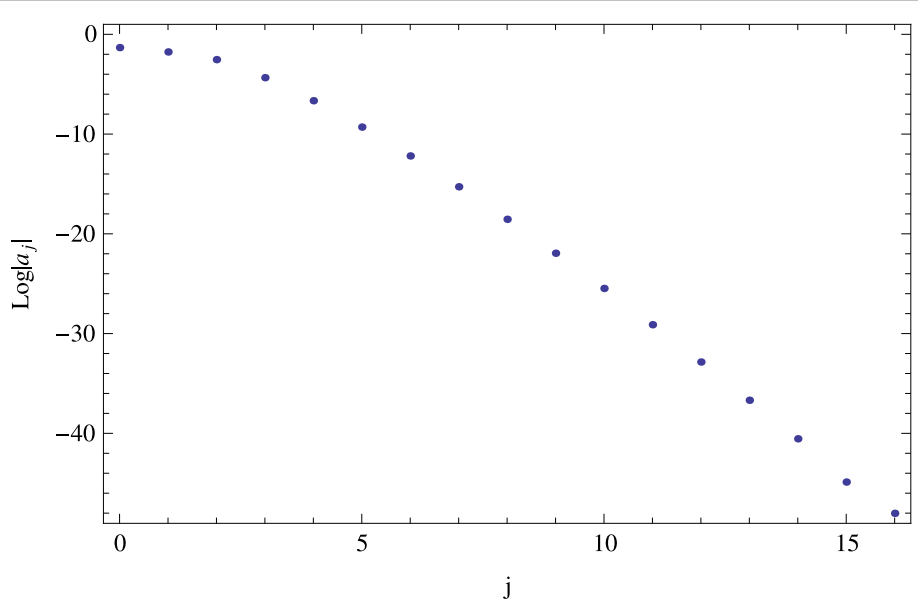

Figure 2 Logarithmic graph of absolute coefficients $\left|a_{j}\right|$ of shifted Legendre polynomials of Example 1.

In Table 2, we compare the errors of the present method with a two-stage order-one Runge-Kutta (RK) method [29], a one-leg $\theta$-method [10,27] with $\theta=0.8$, a variational iteration (VI) method [26], and a shifted Chebyshev operational matrix (SCOM) [30]. The graph of the analytical solution and the approximate solution at $t=5$ for $N=18$ is displayed in Figure 4 to make it easer to compare with analytical solution.

Example 3 Consider the following second-order neutral functional-differential equation with proportional delays [10, 26, 27, 29, 30]:

$$
\left\{\begin{array}{l}
u^{\prime \prime}(x)=\frac{3}{4} u(x)+u\left(\frac{x}{2}\right)+u^{\prime}\left(\frac{x}{2}\right)+\frac{1}{2} u^{\prime \prime}\left(\frac{x}{2}\right)-x^{2}-x+1, \quad x \in[0,1] \\
u(0)=u^{\prime}(0)=0
\end{array}\right.
$$

the exact solution of which is $u(x)=x^{2}$. 


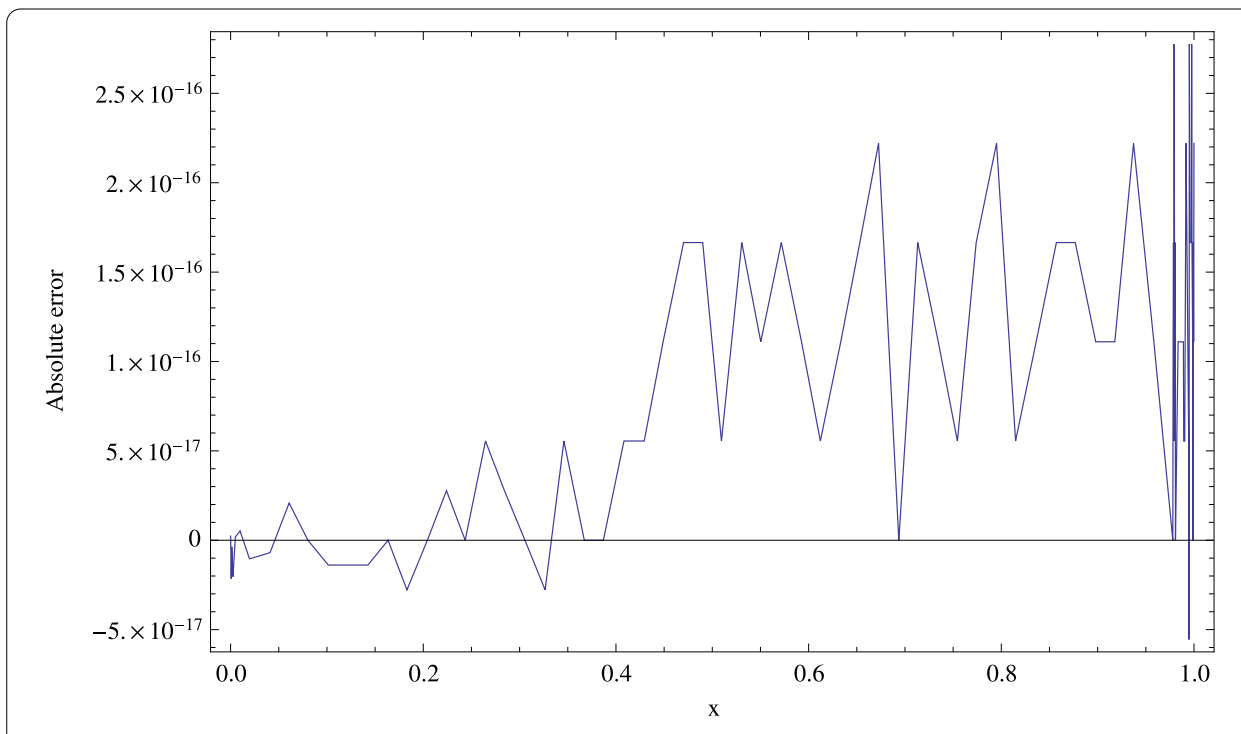

Figure 3 Graph of absolute errors at $N=17$ for Example 1.

Table 2 Absolute errors using SLC method at $N=14$ for Example 2

\begin{tabular}{llllll}
\hline $\boldsymbol{x}$ & $\begin{array}{l}\text { RK } \\
\text { method }\end{array}$ & $\begin{array}{l}\boldsymbol{\theta} \text {-method } \\
\text { with } \boldsymbol{\theta}=\mathbf{0 . 8}\end{array}$ & $\begin{array}{l}\text { VI method } \\
\boldsymbol{m = 8}\end{array}$ & $\begin{array}{l}\text { SCOM } \\
\text { method }\end{array}$ & $\begin{array}{l}\text { SLC method } \\
\mathbf{N = 1 4}\end{array}$ \\
\hline 0.2 & $8.24 \cdot 10^{-4}$ & $8.86 \cdot 10^{-3}$ & $7.08 \cdot 10^{-4}$ & $4.83 \cdot 10^{-11}$ & $1.98 \cdot 10^{-16}$ \\
0.4 & $1.35 \cdot 10^{-3}$ & $2.66 \cdot 10^{-2}$ & $1.29 \cdot 10^{-3}$ & $3.36 \cdot 10^{-11}$ & $8.51 \cdot 10^{-17}$ \\
0.6 & $1.66 \cdot 10^{-3}$ & $4.58 \cdot 10^{-2}$ & $1.76 \cdot 10^{-3}$ & $1.18 \cdot 10^{-11}$ & $1.98 \cdot 10^{-16}$ \\
0.8 & $1.81 \cdot 10^{-3}$ & $6.29 \cdot 10^{-2}$ & $2.15 \cdot 10^{-3}$ & $5.25 \cdot 10^{-11}$ & $2.07 \cdot 10^{-16}$ \\
1.0 & $1.85 \cdot 10^{-3}$ & $7.66 \cdot 10^{-2}$ & $2.47 \cdot 10^{-3}$ & $2.40 \cdot 10^{-12}$ & $4.37 \cdot 10^{-15}$ \\
\hline
\end{tabular}

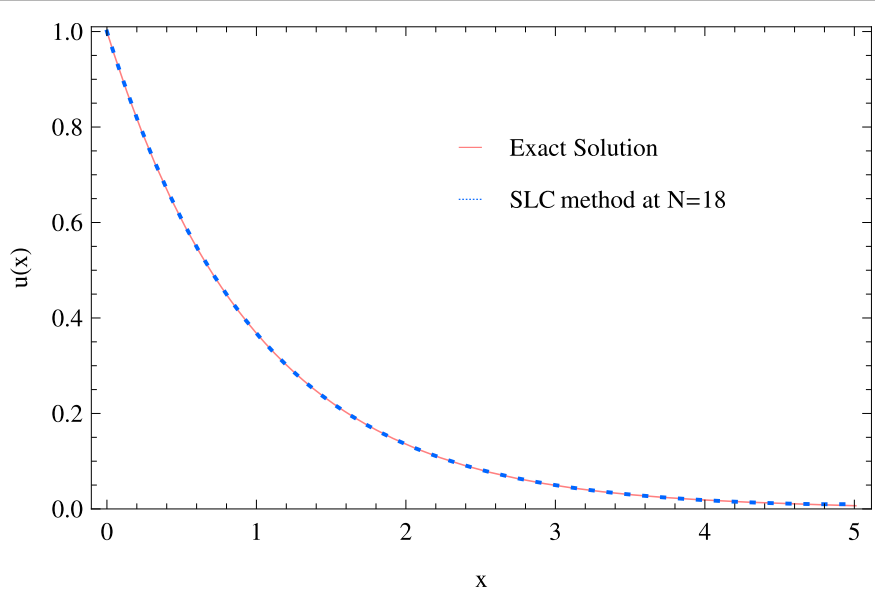

Figure 4 Graph of the exact solution and the approximate solution at $t=5$ and $N=18$ for Example 2 .

In Table 3, we compare the errors of the present method with a two-stage order-one Runge-Kutta (RK) method [29], a one-leg $\theta$-method [10, 27] with $\theta=0.8$, a variational iteration (VI) method [26], and a shifted Chebyshev operational matrix (SCOM) [30]. The graph of the analytical solution and the approximate solution at $t=40$ for $N=8$ is displayed in Figure 5 to make it easer to compare with analytical solution. 
Table 3 Absolute errors using SLC method at $N=10$ for Example 3

\begin{tabular}{llllll}
\hline $\boldsymbol{x}$ & $\begin{array}{l}\text { RK } \\
\text { method }\end{array}$ & $\begin{array}{l}\boldsymbol{\theta} \text {-method } \\
\text { with } \boldsymbol{\theta}=\mathbf{0 . 8}\end{array}$ & $\begin{array}{l}\text { VI method } \\
\boldsymbol{m}=\mathbf{8}\end{array}$ & $\begin{array}{l}\text { SCOM } \\
\text { method }\end{array}$ & $\begin{array}{l}\text { SLC method } \\
\boldsymbol{N = 1 0}\end{array}$ \\
\hline 0.1 & $1.00 \cdot 10^{-3}$ & $6 \cdot 10 \cdot 10^{-3}$ & $1.67 \cdot 10^{-4}$ & $2.08 \cdot 10^{-17}$ & $6.59 \cdot 10^{-17}$ \\
0.2 & $2.02 \cdot 10^{-3}$ & $2.58 \cdot 10^{-2}$ & $7.15 \cdot 10^{-4}$ & $4.16 \cdot 10^{-17}$ & $1.37 \cdot 10^{-17}$ \\
0.3 & $3.07 \cdot 10^{-3}$ & $6.47 \cdot 10^{-2}$ & $1.73 \cdot 10^{-3}$ & $4.16 \cdot 10^{-17}$ & $5.67 \cdot 10^{-18}$ \\
0.4 & $4.17 \cdot 10^{-3}$ & $1.37 \cdot 10^{-1}$ & $3.30 \cdot 10^{-3}$ & $5.55 \cdot 10^{-17}$ & $6.98 \cdot 10^{-17}$ \\
0.5 & $5.34 \cdot 10^{-3}$ & $2.81 \cdot 10^{-1}$ & $5.55 \cdot 10^{-3}$ & $1.11 \cdot 10^{-16}$ & $2.13 \cdot 10^{-17}$ \\
\hline
\end{tabular}

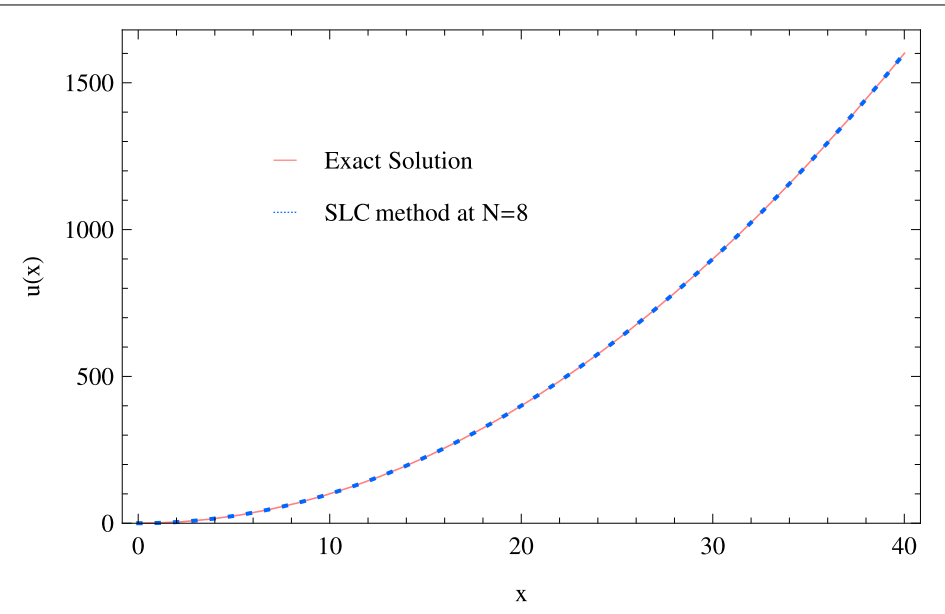

Figure 5 Graph of the exact solution and the approximate solution at $t=40$ and $N=8$ for Example 3 .

Table 4 Absolute errors using SLC method for Example 4

\begin{tabular}{rlrl}
\hline $\boldsymbol{x}$ & SLC mehood & \multicolumn{1}{l}{$\boldsymbol{x}$} & \multicolumn{1}{l}{ SLC mehood } \\
\hline 0.0 & $8.61 \cdot 10^{-18}$ & 0.0 & $3.01 \cdot 10^{-18}$ \\
5.0 & $2.77 \cdot 10^{-17}$ & 10.0 & $1.66 \cdot 10^{-16}$ \\
10.0 & $5.55 \cdot 10^{-17}$ & 20.0 & $3.33 \cdot 10^{-16}$ \\
15.0 & $1.11 \cdot 10^{-16}$ & 30.0 & $1.44 \cdot 10^{-15}$ \\
20.0 & $1.11 \cdot 10^{-16}$ & 40.0 & $4.32 \cdot 10^{-15}$ \\
25.0 & $2.22 \cdot 10^{-16}$ & 50.0 & $1.66 \cdot 10^{-15}$ \\
30.0 & $7.77 \cdot 10^{-16}$ & 60.0 & $3.91 \cdot 10^{-14}$ \\
35.0 & $5.77 \cdot 10^{-15}$ & 70.0 & $7.57 \cdot 10^{-14}$ \\
40.0 & $8.62 \cdot 10^{-13}$ & 80.0 & $4.04 \cdot 10^{-13}$ \\
45.0 & $1.27 \cdot 10^{-10}$ & 90.0 & $1.52 \cdot 10^{-11}$ \\
50.0 & $1.87 \cdot 10^{-8}$ & 100.0 & $1.13 \cdot 10^{-7}$ \\
\hline
\end{tabular}

Example 4 Consider the following third-order neutral functional-differential equation with proportional delays:

$$
\left\{\begin{array}{l}
u^{\prime \prime \prime}(x)=u(x)+u^{\prime}\left(\frac{x}{2}\right)+u^{\prime \prime}\left(\frac{x}{3}\right)+\frac{1}{2} u^{\prime \prime \prime}\left(\frac{x}{4}\right)+f(x), \quad x \in[0,100] \\
u(0)=0, \quad u^{\prime}(0)=\frac{\pi}{100}, \quad u^{\prime \prime}(0)=-\frac{\pi^{2}}{10,000}
\end{array}\right.
$$

and the function $f(x)$ is chosen such that the analytical solution $u(x)=\log \left(1+\sin \left(\frac{\pi x}{100}\right)\right)$.

In Table 4, we list the absolute errors using SLC method in the two intervals $[0,50]$ and $[0,100]$, at $N=20$ and $N=24$ respectively. 


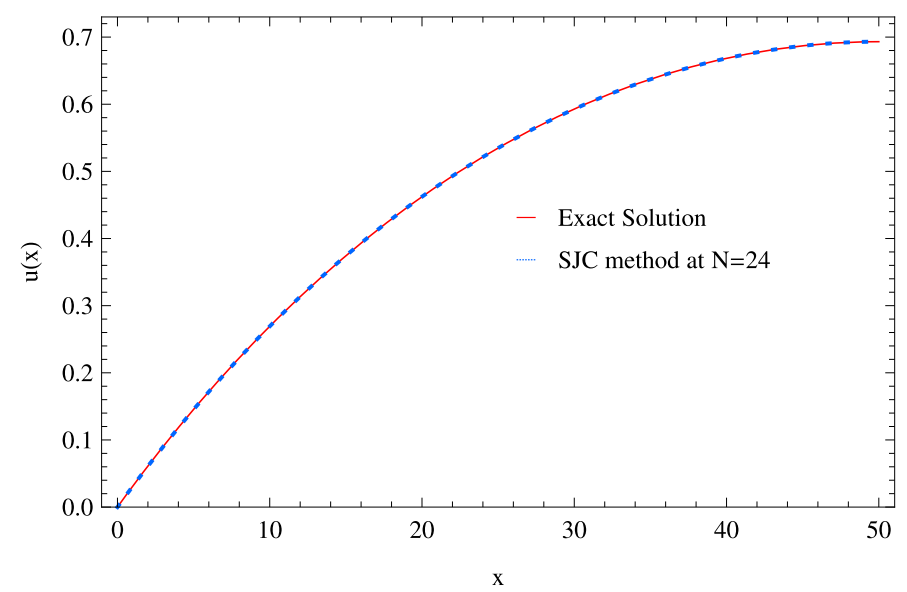

Figure 6 Comparison of the approximate solutions with the exact solution at $t=50$ and $N=24$ for Example 4.

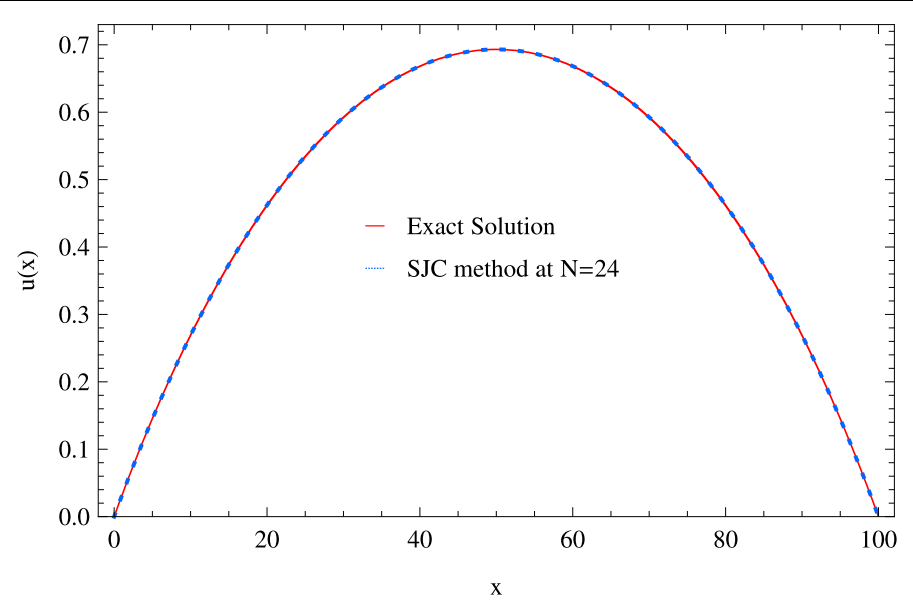

Figure 7 Comparison of the approximate solutions with the exact solution at $t=100$ and $N=24$ for Example 4.

From Table 4, we can see that the approximate solution by the SLC method agrees very well with the exact solution. Figures 6 and 7 display the spectral solution at $N=24$ and the exact one in two long intervals, $[0,50]$ and $[0,100]$, respectively.

\section{Conclusions and future works}

In this paper, we have proposed a new collocation method based on the shifted Legendre polynomials to numerically solve the neutral functional-differential equations with studying the error analysis of the proposed method. The comparison of the obtained results with those based on other methods shows that the present method is a powerful mathematical tool for finding the numerical solutions of such equations. High accuracy in long computational intervals and the stability of the approximated solutions encourage us to apply a similar method for solving other applied mathematics problems (see, for instance, [31]) in the future. 


\section{Competing interests}

The authors declare that they have no competing interests.

\section{Authors' contributions}

The authors have equal contributions to each part of this paper. All the authors read and approved the final manuscript.

\section{Author details}

${ }^{1}$ Department of Mathematics, Faculty of Science, King Abdulaziz University, Jeddah, 21589, Saudi Arabia. ${ }^{2}$ Department of Mathematics, Faculty of Science, Beni-Suef University, Beni-Suef, Egypt. ${ }^{3}$ Department of Mathematics, Faculty of Science, Umm Al-Qura University, Makkah, Saudi Arabia. ${ }^{4}$ Department of Mathematics, Islamic Azad University, Zahedan Branch, Zahedan, Iran.

\section{Acknowledgements}

This study was supported by the Deanship of Scientific Research of King Abdulaziz University. The authors would like to thank the reviewers for their constructive comments and suggestions to improve the quality of the article.

\section{Received: 5 January 2013 Accepted: 28 February 2013 Published: 20 March 2013}

\section{References}

1. Ajello, WG, Freedman, $\mathrm{HI}, \mathrm{Wu}$, J: A model of stage structured population growth with density depended time delay. SIAM J. Appl. Math. 52, 855-869 (1992)

2. Fox, L, Mayers, DF, Ockendon, JA, Tayler, AB: On a functional differential equation. J. Inst. Math. Appl. 8, 271-307 (1971)

3. Ockendon, JR, Tayler, AB: The dynamics of a current collection system for an electric locomotive. Proc. R. Soc. Lond. A $322,447-468$ (1971)

4. Hairer, E, Norsett, SP, Wanner, G: Solving Ordinary Differential Equation I: Nonstiff Problems, 2nd edn. Springer, Berlin (1993)

5. Ghil, M, Zaliapin, I, Thompson, S: A differential delay model of ENSO variability: parametric instability and the distribution of extremes. Nonlinear Process. Geophys. 15, 417-433 (2008)

6. Jackiewicz, Z, Lo, E: The numerical solution of neutral functional differential equations by Adams predictor-corrector methods. Appl. Numer. Math. 8, 477-491 (1991)

7. Dehghan, M, Shakeri, F: The use of the decomposition procedure of Adomian for solving a delay differential equation arising in electrodynamics. Phys. Scr. 78, 11 (2008)

8. Liu, MZ, Li, DS: Properties of analytic solution and numerical solution of multi-pantograph equation. Appl. Math. Comput. 155, 853-871 (2004)

9. Zhao, JJ, Xu, Y, Wang, HX, Liu, MZ: Stability of a class of Runge-Kutta methods for a family of pantograph equations of neutral type. Appl. Math. Comput. 181,1170-1181 (2006)

10. Wang, W, Li, S: On the one-leg $\theta$-methods for solving nonlinear neutral function differential equations. Appl. Math. Comput. 193, 285-301 (2007)

11. Bartoszewski, Z, Kwapisz, M: On the convergence of waveform relaxation methods for differential-functional systems of equations. J. Math. Anal. Appl. 235, 478-496 (1999)

12. Zubik-Kowal, B, Vandewalle, S: Waveform relaxation for functional-differential equations. SIAM J. Sci. Comput. 21, 207-226 (1999)

13. Wu, S, Huang, C: Convergence analysis of waveform relaxation methods for neutral differential-functional systems. J. Comput. Appl. Math. 223, 263-277 (2009)

14. Ishiwata, E, Muroya, Y: Rational approximation method for delay differential equations with proportional delay. Appl. Math. Comput. 187, 741-747 (2007)

15. Ishiwata, E, Muroya, Y, Brunner, H: A super-attainable order in collocation methods for differential equations with proportional delay. Appl. Math. Comput. 198, 227-236 (2008)

16. Cordero, LF, Escalante, R: Segmented Tau approximation for a parametric nonlinear neutral differential equation. Appl. Math. Comput. 190, 866-881 (2007)

17. Tohidi, E, Bhrawy, AH, Erfani, K: A collocation method based on Bernoulli operational matrix for numerical solution of generalized pantograph equation. Appl. Math. Model. 37, 4283-4294 (2013)

18. Wang, ZQ, Wang, LL: A Legendre-Gauss collocation method for nonlinear delay differential equations. Discrete Contin. Dyn. Syst., Ser. B 13, 685-708 (2010)

19. Ali, I, Brunner, H, Tang, T: A spectral method for pantograph-type delay differential equations and its convergence analysis. J. Comput. Math. 27, 254-265 (2009)

20. Ali, I, Brunner, H, Tang, T: Spectral methods for pantograph-type differential and integral equations with multiple delays. Front. Math. China 4, 49-61 (2009)

21. Trif, D: Direct operatorial tau method for pantograph-type equations. Appl. Math. Comput. 219, 2194-2203 (2012)

22. Bhrawy, AH, Alofi, AS: A Jacobi-Gauss collocation method for solving nonlinear Lane-Emden type equations. Commun. Nonlinear Sci. Numer. Simul. 17, 62-70 (2012)

23. Guo, B-Y, Wang, L-L: Jacobi approximations in non-uniformly Jacobi-weighted Sobolev spaces. J. Approx. Theory 128 1-41 (2004)

24. Doha, EH, Bhrawy, AH: An efficient direct solver for multidimensional elliptic robin boundary value problems using a Legendre spectral-Galerkin method. Comput. Math. Appl. 64, 558-571 (2012)

25. LV, X, Gao, Y: The RKHSM for solving neutral functional-differential equations with proportional delays. Math. Methods Appl. Sci. (2012). doi:10.1002/mma.2612

26. Chen, $X$, Wang, L: The variational iteration method for solving a neutral functional-differential equation with proportional delays. Comput. Math. Appl. 59, 2696-2702 (2010)

27. Wang, W, Qin, T, Li, S: Stability of one-leg $\theta$-methods for nonlinear neutral differential equations with proportional delay. Appl. Math. Comput. 213, 177-183 (2009)

28. Wang, W, Zhang, Y, Li, S: Stability of continuous Runge-Kutta-type methods for nonlinear neutral delay-differential equations. Appl. Math. Model. 33, 3319-3329 (2009) 
29. Bellen, A, Zennaro, M: Numerical Methods for Delay Differential Equations, Numerical Mathematics and Scientific Computation. Oxford University Press, New York (2003)

30. Sedaghat, S, Ordokhani, Y, Dehghan, M: Numerical solution of the delay differential equations of pantograph type via Chebyshev polynomials. Commun. Nonlinear Sci. Numer. Simul. 17, 4815-4830 (2012)

31. Bhrawy, AH, Tohidi, E, Soleymani, F: A new Bernoulli matrix method for solving high-order linear and nonlinear Fredholm integro-differential equations with piecewise intervals. Appl. Math. Comput. 219, $482-497$ (2012)

doi:10.1186/1687-1847-2013-63

Cite this article as: Bhrawy et al.: A Legendre-Gauss collocation method for neutral functional-differential equations with proportional delays. Advances in Difference Equations 2013 2013:63.

Submit your manuscript to a SpringerOpen ${ }^{\circ}$ journal and benefit from:

- Convenient online submission

Rigorous peer review

- Immediate publication on acceptance

- Open access: articles freely available online

- High visibility within the field

- Retaining the copyright to your article

Submit your next manuscript at $\boldsymbol{s p r i n g e r o p e n . c o m ~}$ 